\title{
Physical activity, multimorbidity, and life expectancy: a UK Biobank longitudinal study
}

Yogini V. Chudasama ${ }^{1,2^{*}}$ (D), Kamlesh K. Khunti ${ }^{1,2}$, Francesco Zaccardi ${ }^{1}$, Alex V. Rowlands ${ }^{1,3,4}$, Thomas Yates ${ }^{1,3}$, Clare L. Gillies ${ }^{1}$, Melanie J. Davies ${ }^{1,3}$ and Nafeesa N. Dhalwani ${ }^{1,2,5}$

\begin{abstract}
Background: Multimorbidity is an emerging public health priority. Physical activity (PA) is recommended as one of the main lifestyle behaviours, yet the benefits of PA for people with multimorbidity are unclear. We assessed the benefits of PA on mortality and life expectancy in people with and without multimorbidity.

Methods: Using the UK Biobank dataset, we extracted data on 36 chronic conditions and defined multimorbidity as (a) 2 or more conditions, (b) 2 or more conditions combined with self-reported overall health, and (c) 2 or more top-10 most common comorbidities. Leisure-time PA (LTPA) and total PA (TPA) were measured by questionnaire and categorised as low (<600 metabolic equivalent (MET)-min/week), moderate (600 to $<3000$ MET-min/week), and high ( $\geq 3000$ MET-min/week), while objectively assessed PA was assessed by wrist-worn accelerometer and categorised as low (4 min/day), moderate (10 min/day), and high (22 min/day) walking at brisk pace. Survival models were applied to calculate adjusted hazard ratios (HRs) and predict life expectancy differences.

Results: 491,939 individuals (96,622 with 2 or more conditions) had a median follow-up of 7.0 (IQR 6.3-7.6) years. Compared to low LTPA, for participants with multimorbidity, HR for mortality was 0.75 ( $95 \% \mathrm{Cl} 0.70-0.80)$ and $0.65(0.56-0.75)$ in moderate and high LTPA groups, respectively. This finding was consistent when using TPA measures. Using objective PA, HRs were 0.49 (0.29-0.80) and 0.29 (0.13-0.61) in the moderate and high PA groups, respectively. These findings were similar for participants without multimorbidity. In participants with multimorbidity, at the age of 45 years, moderate and high LTPA were associated with an average of $3.12(95 \% \mathrm{Cl} 2.53,3.71)$ and 3.55 $(2.34,4.77)$ additional life years, respectively, compared to low LTPA; in participants without multimorbidity, corresponding figures were $1.95(1.59,2.31)$ and $1.85(1.19,2.50)$. Similar results were found with TPA. For objective PA, moderate and high levels were associated with $3.60(-0.60,7.79)$ and $5.32(-0.47,11.11)$ life years gained compared to low PA for those with multimorbidity and $3.88(1.79,6.00)$ and $4.51(2.15,6.88)$ life years gained in those without. Results were consistent when using other definitions of multimorbidity.
\end{abstract}

Conclusions: There was an inverse dose-response association between PA and mortality. A moderate exercise is associated with a longer life expectancy, also in individuals with multimorbidity.

Keywords: Multimorbidity, Co-morbidity, Physical activity, Life expectancy, Mortality, UK Biobank

\footnotetext{
* Correspondence: yc244@leicester.ac.uk

${ }^{1}$ Diabetes Research Centre, Leicester General Hospital, University of Leicester, Leicester LE5 4PW, UK

${ }^{2}$ National Institute for Health Research (NIHR) Collaboration for Leadership in

Applied Health Research and Care - East Midlands (CLAHRC-EM), Leicester

Diabetes Centre, Leicester LE5 4PW, UK

Full list of author information is available at the end of the article
}

(c) The Author(s). 2019 Open Access This article is distributed under the terms of the Creative Commons Attribution 4.0 International License (http://creativecommons.org/licenses/by/4.0/), which permits unrestricted use, distribution, and reproduction in any medium, provided you give appropriate credit to the original author(s) and the source, provide a link to the Creative Commons license, and indicate if changes were made. The Creative Commons Public Domain Dedication waiver (http://creativecommons.org/publicdomain/zero/1.0/) applies to the data made available in this article, unless otherwise stated. 


\section{Background}

The average life expectancy continues to increase in most of developed countries; however, this longevity does not necessarily translate in better health [1]. The ageing phenomenon has led to a substantial increase in chronic conditions, which consequently result in a rising prevalence of multimorbidity, most commonly described as the presence of two or more long-term conditions [2-4]. Multimorbidity has been linked to poor prognosis, lower quality of life [5], increased health care costs, and the risk of premature death [6]. Management of multimorbidity is a complex process, which recently has become an emerging priority for public health care professionals and health care systems [7-9]. Physical activity (PA) has been recommended as one of the main lifestyle behaviours in the management of several chronic conditions worldwide [10-12]. Yet, it is not clear whether and to what extent the benefits of PA apply to people with multimorbidity [9].

To our knowledge, only two studies to date have assessed the relationship between PA and mortality in people with multimorbidity, with inconsistent findings. The first study showed that, in people with multimorbidity, high PA levels were related to a significantly lower risk of mortality, compared to being physically inactive [13]. Conversely, the second study demonstrated that the risk of mortality was only significantly reduced when engaging in extremely high levels of PA ( $\geq 8000$ moderate-vigorous PA MET-min/month), which may not be feasible for individuals with multimorbidity [14]. Moreover, both studies used self-reported PA measures which have several limitations [15]. For this reason, there is a need for accurate and reliable measures of PA $[16,17]$. Objective PA is capable of capturing precise estimates of energy expenditure at different activity intensities, in particular recording light PA [18]. Yet, to date, no study has examined the association between objective PA and mortality in people with multimorbidity. Furthermore, the life expectancy according to PA levels and multimorbidity status remains not well determined.

We aimed to investigate the association between PA and all-cause mortality and to estimate the effects of PA on life expectancy in people with and without multimorbidity, using both subjective and objective measures of PA in a large contemporary cohort from the UK.

\section{Methods}

\section{Data source and study population}

This research has been conducted using the UK Biobank Resource (Application Number 14146). UK Biobank is a prospective study designed to improve the prevention, diagnosis, and treatment of chronic diseases in people aged between 38 and 73 years recruited from 22 sites across the UK. This study included 502,611 participants with baseline measures collected between 2006 and 2010 [19], and data have since been linked to hospital and mortality records. A sub-sample of 103,704 also completed objective measures of PA between 2013 and 2015 [20]. Participants gave written informed consent prior to data collection, and ethical approval was obtained by the North-West Research Ethics Committee [21]. To minimise reverse causality (i.e. undiagnosed, subclinical disease(s) leading to lower exercise and mortality), we excluded participants who died within 2 years followup from baseline assessment $(n=2416)$ and also excluded participants with no data for the self-reported PA $(n=8256)$, leaving 491,939 participants for analysis.

\section{Defining multimorbidity}

The UK Biobank collected self-reported medical information based on physician diagnosis [22]. Three sources of selecting long-term cardiovascular, non-cardiovascular, or mental health conditions were used [23]. The first included conditions from the quality and outcomes framework (QoF) which report the most common diseases in the UK [24]; the second was from a large UK-based study, containing 40 of the recommended core disorders for any multimorbidity measure [7]; and the last was from a systematic review on multimorbidity indices that included 17 conditions [25]. Based on these sources, we selected a total of 36 chronic conditions. Participants with two or more of these 36 chronic conditions were classified as having multimorbidity (Additional file 1: Methods S1).

\section{Mortality}

Mortality data were obtained from the National Health Service (NHS) Information Centre for participants from England and Wales and the NHS Central Register for participants from Scotland. Data for survivors were censored on 31 January 2016 for England and Wales and 30 November 2015 for Scotland.

\section{Physical activity}

PA was measured both subjectively and objectively. Two questionnaires were used for subjectively reported (self-reported) PA: (1) leisure-time PA (LTPA), which included five activities undertaken in the last 4 weeks-walking, light DIY (do-it-yourself), heavy DIY, strenuous sports, and other exercises, and (2) a modified version of the International Physical Activity Questionnaire, which assessed total physical activity (TPA) [26], including walking, moderate, and vigorous PA performed over the last 7 days. We categorised participants by three mutually exclusive groups: low (<600 metabolic equivalent (MET)-min/week), moderate (600 to $<3000$ MET-min/week), and high $(\geq 3000$ MET-min/week) PA based on a standard scoring criteria [26]; the threshold at $600 \mathrm{MET}-\mathrm{min} /$ week is equivalent to reaching the recommended guidelines (150 min per week) 
for moderate-intensity PA. Objective PA was measured using the Axivity AX3 wrist-worn triaxial accelerometer (Axivity Ltd., Newcastle, UK), where participants were requested to wear the monitor continuously for seven consecutive days [20]. We excluded participants with less than 3 days of wear data, without wear data in each 1-h period of the 24-h cycle, or with failed accelerometer calibration. We used the overall acceleration average, since it measured the total time spent across all levels of PA intensity. Within the population distribution, we categorised the overall acceleration average by tertiles and based the estimates on the median value. For ease of interpretation, based on the results of a laboratory calibration study [27], we described PA of each tertile in terms of walking at a brisk pace for exercise' of low ( $4 \mathrm{~min} /$ day), moderate $(10 \mathrm{~min} /$ day $)$, and high (22 min/day) (Additional file 1: Methods S2).

\section{Other covariates}

Other relevant covariates, including sociodemographic (age, sex, ethnicity, education level, employment status, and socioeconomic status (Townsend deprivation index)) and lifestyle (smoking status, body mass index, alcohol consumption, fruit and vegetable portions, oily fish, non-oily fish, processed meat, red meat intake, and sedentary behaviour) factors, were also extracted (Additional file 1: Methods S3).

\section{Statistical analysis}

We estimated the prevalence of multimorbidity, with the pattern of comorbidities illustrated using heat maps. Baseline characteristics stratified by multimorbidity status were summarised using numbers, proportions, and medians with interquartile range (IQR). We created an additional category for each sociodemographic and lifestyle covariate to account for missing data. For each PA measure, participants with complete PA data were included in the analyses, i.e. complete-case analysis for LTPA $(n=488,574)$, TPA $(n=454,639)$, and objective PA $(n=95,616)$. Spearman's rank correlation was used to assess the agreement between the two continuous subjective PA measures and between the continuous subjective and objective PA measures. We used Cox proportional hazards regression, with time since baseline assessment as the start of follow-up, to model the association between PA and all-cause mortality in individuals with and without multimorbidity. Hazard ratios (HR) and corresponding 95\% confidence intervals (95\% CI) were calculated, and Schoenfeld's residuals were used to verify the proportional hazards assumption. Four incremental models were fitted: model 1-unadjusted; model 2-adjusted for age and sex; model 3-additionally adjusted for sociodemographic factors; and model 4-additionally adjusted for lifestyle factors. The lowest PA group was the reference category. The calculation of years of life lost (i.e. difference in life expectancy) involved a two-step process using flexible parametric survival models with age as time scale. First, residual life expectancy was estimated as the area under the survival curve up to 100 years old, conditional on surviving at ages 45 to 100 years old (1-year intervals). Second, the differences in years of life were calculated as the difference between the areas under two survival curves [28], i.e. the difference between life expectancy for the moderate or high PA group with the reference of the low PA group by multimorbidity status. To calculate life expectancy, proportional hazard survival analyses were conducted with the stpm 2 command which uses restricted cubic splines to model the baseline cumulative hazard $[29,30]$. Statistical codes are available at GitHub yc244.

\section{Sensitivity analysis}

We grouped self-reported PA measures in tertiles and calculated HR (95\% CI) and differences in residual life expectancy. Additionally, the PA measures were investigated as continuous variables using a $\log$ base of 1.1 (i.e. $\log 1.1$ (total PA)) to represent a 10\% increase in total PA. Due to the time difference between the baseline and objective PA measurements, we further analysed the association between objective PA and mortality taking the time at objective PA assessment as the start of the follow-up. Model 1 was unadjusted, model 2 was adjusted for age at objective PA measurement and sex, and model 3 was further adjusted for ethnicity and socioeconomic status. Adjustments for other lifestyle factors were not possible as they may have changed between the baseline measurement and objective PA measurement. We also recalculated the HR and corresponding 95\% CI for the association between objective PA and mortality; instead of the tertiles being based on the overall acceleration, we based the tertiles on the time accumulated above $250 \mathrm{mg}$ of total acceleration (Additional file 1: Methods S2). Since we categorised multimorbidity as any two or more chronic condition combinations, it is possible that combinations can include two "high-impact" (e.g. diabetes and heart failure) or "low-impact" (e.g. hypertension and asthma) conditions. Therefore, we performed two additional analyses to account for the severity of the conditions. Firstly, we used participants' self-reported overall health rating (UK Biobank Data-Field 2178), as a proxy of severity, and categorised participants into four groups: good health without multimorbidity (reference), poor health without multimorbidity, good health with multimorbidity, and poor health with multimorbidity; participants with missing health rating were excluded from analyses $(n=$ 2324). Secondly, we used the 10 most common comorbidities [31] and categorised participants into two groups: with and without top-10 comorbidity. We repeated the analyses using the three PA exposures. A table summarises main and sensitivity analyses (Additional file 1: Methods S5). All analyses were performed using Stata 14 MP. 


\section{Results}

\section{Participant characteristics}

The five most prevalent chronic conditions in 491,939 participants were hypertension (25.9\%), asthma (11.6\%), cancer (8.2\%), depression (5.6\%), and diabetes (4.2\%). The most prevalent comorbidities include chronic kidney disease and hypertension (70\%), diabetes and hypertension (64\%), and myocardial infarction and angina (42\%) (Fig. 1). The total number of conditions ranged from 0 to 10 , where the overall prevalence of multimorbidity ( $\geq 2$ chronic conditions) was $19.6 \%(n=96,622)$. Most participants were white $(94.3 \%)$, with a median (IQR) age of 58 years (50-63). Participants with multimorbidity were mainly older compared to participants without (> 60 years: $52 \%$ vs. $35 \%$, respectively), lived in deprived areas (most deprived quintile: 24\% vs. 19\%, respectively), were overweight or obese ( $77 \%$ vs. $64 \%$, respectively), had slightly lower consumption of alcohol (33\% vs. $38 \%$ consuming $\geq 14$ units/week, respectively), and spent more time in sedentary behaviour (26\% vs. $20 \%$ spending $\geq 6 \mathrm{~h}$, respectively) (Table 1 ). The two self-reported PA exposures were positively correlated (Pearson's $r=0.41 ; P<0.001$ ) and between both subjective and objective PA measures (Pearson's $r=0.19 ; P<0.001$ ). When categorised into low, moderate, and high PA levels, more than double of the participants with multimorbidity were in the low PA group than in the high PA group (objective PA $47 \%$ vs. $22 \%$, respectively). We found similar baseline characteristics when accounting for the severity of the conditions, top-10 comorbidity,

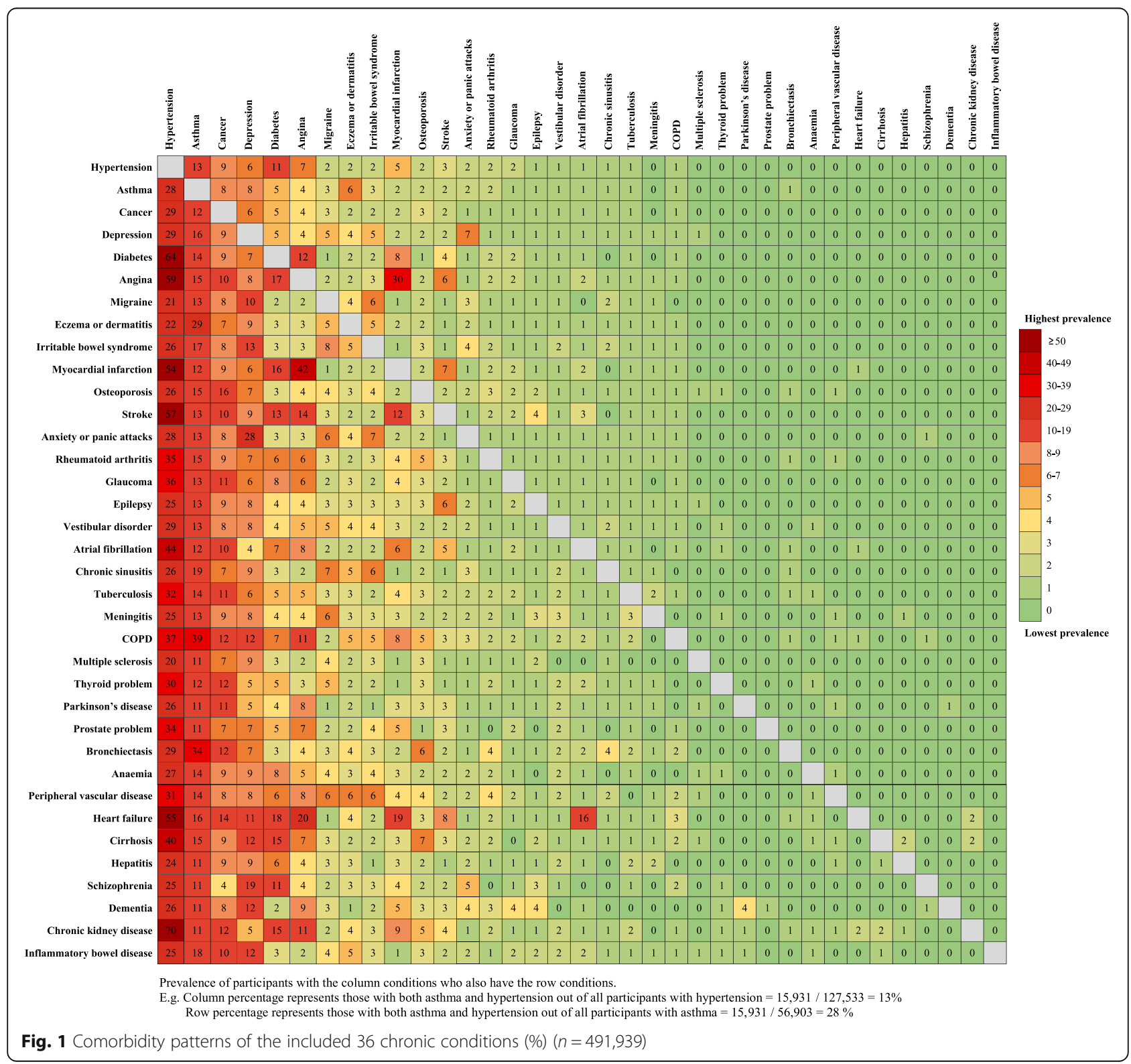


Table 1 Participant characteristics by multimorbidity status (2 or more conditions)

\begin{tabular}{|c|c|c|c|c|}
\hline \multirow[t]{2}{*}{ Characteristics } & \multirow{2}{*}{$\begin{array}{l}\text { All participants } \\
(N=491,939)\end{array}$} & \multicolumn{3}{|l|}{ Multimorbidity status } \\
\hline & & With multimorbidity $(n=96,622)$ & Without multimorbidity $(n=395,317)$ & $P$ value \\
\hline \multicolumn{5}{|l|}{ Sex } \\
\hline Female & $267,883(54.5)$ & $51,801(53.6)$ & $216,082(54.7)$ & \\
\hline Male & $224,056(45.5)$ & $44,821(46.4)$ & $179,235(45.3)$ & $<0.001$ \\
\hline Age, years (median [IQR]) & $58[50-63]$ & $61[54-65]$ & $57[49-63]$ & $<0.001$ \\
\hline \multicolumn{5}{|l|}{ Age categories } \\
\hline$\leq 50$ & $129,832(26.4)$ & $14,787(15.3)$ & $115,045(29.1)$ & \\
\hline $51-60$ & $173,712(35.3)$ & $31,659(32.8)$ & $142,053(35.9)$ & \\
\hline$>60$ & $188,395(38.3)$ & $50,176(51.9)$ & $138,219(35.0)$ & $<0.001$ \\
\hline \multicolumn{5}{|l|}{ Ethnicity } \\
\hline White & $463,804(94.3)$ & $91,013(94.2)$ & $372,791(94.3)$ & \\
\hline Non-white & $26,401(5.4)$ & $5222(5.4)$ & $21,179(5.4)$ & \\
\hline Missing & $1734(0.4)$ & $387(0.4)$ & $1347(0.3)$ & 0.016 \\
\hline \multicolumn{5}{|l|}{ Socioeconomic status } \\
\hline 1st quintile (least deprived) & $99,178(20.2)$ & $17,163(17.8)$ & $82,015(20.7)$ & \\
\hline 2nd quintile & $98,418(20.0)$ & $17,753(18.4)$ & $80,665(20.4)$ & \\
\hline 3rd quintile & $98,401(20.0)$ & $18,392(19.0)$ & $80,009(20.2)$ & \\
\hline 4th quintile & $98,325(20.0)$ & $19,647(20.3)$ & $78,678(19.9)$ & \\
\hline 5th quintile (most deprived) & $97,001(19.7)$ & $23,543(24.4)$ & $73,458(18.6)$ & \\
\hline Missing & $616(0.1)$ & $124(0.1)$ & $492(0.1)$ & $<0.001$ \\
\hline \multicolumn{5}{|l|}{ Education level } \\
\hline College or university degree & $160,218(32.6)$ & $25,630(26.5)$ & $134,588(34.0)$ & \\
\hline A/AS level or equivalent & $54,924(11.2)$ & $9791(10.1)$ & $45,133(11.4)$ & \\
\hline O levels/GCSEs or equivalent & $130,813(26.6)$ & $24,154(25.0)$ & $106,659(27.0)$ & \\
\hline Other (e.g. NVQ, nursing, missing) & $145,984(29.7)$ & $37,047(38.3)$ & $108,937(27.6)$ & $<0.001$ \\
\hline \multicolumn{5}{|l|}{ Employment status } \\
\hline Working & $283,151(57.6)$ & $39,205(40.6)$ & $243,946(61.7)$ & \\
\hline Retired & $163,238(33.2)$ & $43,889(45.4)$ & $119,349(30.2)$ & \\
\hline Unemployed & $8112(1.6)$ & $1635(1.7)$ & $6477(1.6)$ & \\
\hline Other (student, volunteer/missing) & $37,438(7.6)$ & $11,893(12.3)$ & $25,545(6.5)$ & $<0.001$ \\
\hline \multicolumn{5}{|l|}{ BMl categories, $\mathrm{kg} / \mathrm{m}^{2}$} \\
\hline Underweight (< 18.5) & $2515(0.5)$ & $431(0.4)$ & $2084(0.5)$ & \\
\hline Normal weight (18.5-24.9) & $159,596(32.4)$ & $21,591(22.3)$ & $138,008(34.9)$ & \\
\hline Overweight (25.0-29.9) & $208,375(42.4)$ & $38,546(39.9)$ & $169,829(43.0)$ & \\
\hline Obese $(\geq 30.0)$ & $119,081(24.2)$ & $35,450(36.7)$ & $83,631(21.2)$ & \\
\hline Missing & $2372(0.5)$ & $604(0.6)$ & $1768(0.4)$ & $<0.001$ \\
\hline \multicolumn{5}{|l|}{ Smoking status } \\
\hline Never & $269,050(54.7)$ & $46,543(48.2)$ & $222,507(56.3)$ & \\
\hline Former & $169,693(34.5)$ & $39,068(40.4)$ & $130,625(33.0)$ & \\
\hline Current & $51,342(10.4)$ & $10,524(10.9)$ & $40,818(10.3)$ & \\
\hline Missing & $1854(0.4)$ & $487(0.5)$ & $1367(0.3)$ & $<0.001$ \\
\hline \multicolumn{5}{|l|}{ Alcohol consumption } \\
\hline Never or < 14 units/week & $308,029(62.6)$ & $64,377(66.6)$ & $243,652(61.6)$ & \\
\hline Excess $\geq 14$ units/week & $183,249(37.3)$ & $32,088(33.2)$ & $151,161(38.2)$ & \\
\hline Missing & $661(0.1)$ & $157(0.2)$ & $504(0.1)$ & $<0.001$ \\
\hline
\end{tabular}


Table 1 Participant characteristics by multimorbidity status (2 or more conditions) (Continued)

\begin{tabular}{|c|c|c|c|c|}
\hline \multirow[t]{2}{*}{ Characteristics } & \multirow{2}{*}{$\begin{array}{l}\text { All participants } \\
(N=491,939)\end{array}$} & \multicolumn{3}{|l|}{ Multimorbidity status } \\
\hline & & With multimorbidity $(n=96,622)$ & Without multimorbidity $(n=395,317)$ & $P$ value \\
\hline \multicolumn{5}{|l|}{ Meet fruit/vegetable guidelines } \\
\hline No & $304,709(61.9)$ & $58,682(60.7)$ & $246,027(62.2)$ & \\
\hline Yes & $186,628(37.9)$ & $37,818(39.1)$ & $148,810(37.6)$ & \\
\hline Missing & $602(0.1)$ & $122(0.1)$ & $480(0.1)$ & $<0.001$ \\
\hline \multicolumn{5}{|l|}{ Oily fish ( $\geq 1 /$ week) } \\
\hline No & $218,092(44.3)$ & $40,954(42.4)$ & $177,138(44.8)$ & \\
\hline Yes & $273,627(55.6)$ & $55,636(57.6)$ & $217,981(55.1)$ & \\
\hline Missing & $230(0.0)$ & $32(0.0)$ & $198(0.1)$ & $<0.001$ \\
\hline \multicolumn{5}{|l|}{ Non-oily fish ( $\geq 1 /$ week) } \\
\hline No & $167,300(34.0)$ & $31,915(33.0)$ & $135,385(34.2)$ & \\
\hline Yes & $324,435(66.0)$ & $64,682(66.9)$ & $259,753(65.7)$ & \\
\hline Missing & $204(0.0)$ & $25(0.0)$ & $179(0.0)$ & $<0.001$ \\
\hline \multicolumn{5}{|l|}{ Processed meat ( $\geq 2 /$ week) } \\
\hline No & $339,723(69.1)$ & $65,198(67.5)$ & $274,525(69.4)$ & \\
\hline Yes & $152,022(30.9)$ & $31,394(32.5)$ & $120,628(30.5)$ & \\
\hline Missing & $194(0.0)$ & $30(0.0)$ & $164(0.0)$ & $<0.001$ \\
\hline \multicolumn{5}{|l|}{ Red meat ( $\geq 2 /$ week) } \\
\hline No & $418,282(85.0)$ & $81,011(83.8)$ & $337,271(85.3)$ & \\
\hline Yes & $73,542(14.9)$ & $15,598(16.1)$ & $57,944(14.7)$ & \\
\hline Missing & $115(0.0)$ & $13(0.0)$ & $102(0.0)$ & $<0.001$ \\
\hline \multicolumn{5}{|l|}{ Sedentary behaviour, hours } \\
\hline Low $(<4)$ & $222,081(45.1)$ & $38,314(39.7)$ & $183,767(46.5)$ & \\
\hline Moderate (4-6) & $166,435(33.8)$ & $33,326(34.5)$ & $133,109(33.7)$ & \\
\hline High ( $\geq 6)$ & $102,911(20.9)$ & $24,839(25.7)$ & $78,072(19.7)$ & \\
\hline Missing & $512(0.1)$ & $143(0.1)$ & $369(0.1)$ & $<0.001$ \\
\hline LTPA, MET-min/week, (median [IQR]) & 619 [200-1353] & $464[118-1154]$ & $656[228-1406]$ & $<0.001$ \\
\hline \multicolumn{5}{|l|}{ LTPA categories, MET-min/week } \\
\hline Low $(<600)$ & 238,971 (48.6) & $53,658(55.5)$ & $185,313(46.9)$ & \\
\hline Moderate (600 to < 3000) & $215,517(43.8)$ & $36,760(38.1)$ & $178,757(45.2)$ & \\
\hline High $(\geq 3000)$ & $34,086(6.9)$ & $5199(5.4)$ & $28,887(7.3)$ & \\
\hline Missing & $3365(0.7)$ & $1005(1.0)$ & $2360(0.6)$ & $<0.001$ \\
\hline TPA MET-min/week, (median [IQR]) & 1655 [737-3360] & 1440 [636-3093] & 1706 [780-3430] & $<0.001$ \\
\hline \multicolumn{5}{|l|}{ TPA categories, MET-min/week } \\
\hline Low $(<600)$ & $90,698(18.4)$ & $20,823(21.6)$ & $69,875(17.7)$ & \\
\hline Moderate (600 to < 3000) & $232,856(47.3)$ & $43,120(44.6)$ & $189,736(48.0)$ & \\
\hline High $(\geq 3000)$ & $131,085(26.7)$ & $22,655(23.5)$ & $108,430(27.4)$ & \\
\hline Missing & $37,300(7.6)$ & $10,024(10.4)$ & $27,276(6.9)$ & $<0.001$ \\
\hline Objective mg, (median [IQR] $)^{\mathrm{a}}$ & $27.1[22.4-32.5]$ & $24.5[20.1-29.7]$ & $27.6[22.9-33.0]$ & $<0.001$ \\
\hline \multicolumn{5}{|c|}{ Objective categories, min/day of brisk walking ${ }^{a}$} \\
\hline Low (4) & $31,919(33.4)$ & $7369(47.2)$ & $24,550(30.7)$ & \\
\hline Moderate (10) & $31,871(33.3)$ & $4757(30.5)$ & $27,114(33.9)$ & \\
\hline High (22) & $21,826(33.3)$ & $3481(22.3)$ & $28,345(35.4)$ & $<0.001$ \\
\hline
\end{tabular}

Shown are numbers (\%) unless stated otherwise. $P$ value indicates the difference between categories (Chi-square test); continuous data (Wilcoxon ranksum test). A/AS level or equivalent $=$ higher school certificate; $\mathrm{O}$ levels/GCSEs $=$ school certificate

NVQ National Vocational Qualification, BMI body mass index, LTPA leisure-time physical activity questionnaire, TPA total physical activity questionnaire, MET metabolic equivalent of task, $m g$ milli-gravitational units

${ }^{a}$ Data taken at the time of objective physical activity measurements 
and the subsample of participants who undertook the objective PA. Participants who completed the objective PA measurements were generally from higher affluent areas, more educated, in employment, and less likely to be current smokers or obese compared to non-participants (Additional file 2: Tables S1, S2, S3, S4, S5, S6, S7, and S8, Figure S1).

\section{Subjective physical activity and all-cause mortality}

The median follow-up was 7.0 [range 2.0-9.1] years with 11,479 recorded deaths, of which $38 \%(n=4399)$ occurred in participants with multimorbidity. Self-reported PA showed an inverse dose-response association with mortality for all participants, independent of multimorbidity status and adjustments for confounders (Fig. 2). Using LTPA, in participants with multimorbidity, the risk of mortality was $25 \%$ lower in the moderate PA group (HR 0.75, 95\% CI 0.70-0.80) and 35\% lower in the high PA group (HR 0.65, 95\% CI 0.56-0.75), compared to the low PA group in the fully adjusted model; corresponding figures for participants without multimorbidity were 20\% (HR 0.80, 95\% CI 0.76-0.85) and 25\% (HR 0.75 , 95\% CI 0.68-0.82), respectively. Using TPA, in participants with multimorbidity, the risk of mortality was $14 \%$ lower in the moderate PA group (HR 0.86, 95\% CI $0.79-0.93$ ) and $22 \%$ lower in the high PA group (HR 0.78, 95\% CI 0.71-0.85), compared to the low PA group, in the fully adjusted model; corresponding figures for participants without multimorbidity were $17 \%$ (HR 0.83 , 95\% CI $0.78-0.89$ ) and 19\% (HR 0.81, 95\% CI $0.76-$ 0.87 ), respectively. Results were consistent in sensitivity analyses with (1) LTPA and TPA categorised into tertiles, (2) continuously measured (log-transformed) PA, (3) alternative definitions of multimorbidity, accounting for overall health rating, or top-10 comorbidity (Additional file 2: Tables S10, S11 and 12, Figure S2).

\section{Objective physical activity and all-cause mortality}

95,616 participants completed the objective PA measurements, of which $16.3 \%(n=15,607)$ participants had multimorbidity. Using time since baseline assessment as the start of the follow-up, the median follow-up was 6.9 (range 3.8-9.1) years with 306 participant (0.3\%) deaths. The amount of time spent walking 'at a brisk pace for exercise' (> $250 \mathrm{mg}$ ) was 4,10 , and $22 \mathrm{~min} /$ day in the low, moderate, and high volume PA groups. In the fully adjusted model, the risk of mortality in participants with multimorbidity was $51 \%$ lower in the moderate PA group (HR 0.49, 95\% CI 0.29-0.80) and 71\% lower in the high PA group, (HR 0.29, 95\% CI 0.130.61) compared to the low PA group (Fig. 3). In participants without multimorbidity, there was a $60 \%$ (HR $0.40,95 \%$ CI $0.29-0.57)$ and $71 \%$ (HR $0.29,95 \%$ CI 0.19-0.46) lower risk of mortality in the moderate and high PA group respectively, compared to low PA (Fig. 2). Results were consistent in sensitivity analyses considering the following: (1) the objective PA assessment as the start of the follow-up (median follow-up was 1.23 (IQR 0.70, 1.72) years), (2) tertiles of time accumulated above $250 \mathrm{mg}$ of total acceleration, (3) log-transformed objective PA, (4) alternative definitions of multimorbidity, accounting for overall health rating, or top-10 conditions (see Additional file 2: Tables S10, S11, S12 and S13, Figure S3).

\section{Life expectancy \\ Multimorbidity}

At the age of 45 years, participants with multimorbidity had, on average, a life expectancy which was 5.15 (95\% CI $4.78,5.14$ ) lower than participants without multimorbidity; the corresponding figure at the age of 65 years was 4.54 (95\% CI 4.21, 4.87) years (Fig. 3). In sensitivity analyses, when accounting for the severity of the conditions, at 45 years, participants with poor health and multimorbidity had 9.00 (95\% CI 8.47, 9.53) years lower life expectancy compared to those with good health without multimorbidity, while participants with top-10 comorbidity had 6.53 (95\% CI 6.01, 7.05) years lower life expectancy compared to those without.

\section{Self-reported physical activity}

Using LTPA, in participants aged 45 years with multimorbidity, moderate and high PA were associated with an average of $3.12(95 \%$ CI $2.53,3.71)$ and 3.55 (95\% CI $2.34,4.77$ ) additional life years gained, respectively, compared to low PA; corresponding figures in those without multimorbidity were 1.95 (95\% CI $1.59,2.31)$ and 1.85 (95\% CI 1.19, 2.50) additional life years gained, respectively (Fig. 4). The effects of increased PA were lower in older participants such that the benefit was found to decrease with age. Using TPA, in participants aged 45 years with multimorbidity, moderate and high PA were associated with 2.04 (95\% CI 1.35, 2.74) and 2.92 (95\% CI $2.12,3.73)$ additional life years gained, respectively, compared to low PA; corresponding figures in those without multimorbidity in the same group were 1.79 (95\% CI $1.32,2.27)$ and $1.76(95 \%$ CI $1.25,2.27)$ additional life years, respectively (Fig. 4). Results were similar in sensitivity analyses using tertiles rather than PA categories, considering the severity of the conditions or top-10 comorbidity (Additional file 2: Tables S9, S11, and S12).

\section{Objective physical activity}

Using objective PA, in participants with multimorbidity aged 45 years, moderate and high PA groups were associated with $3.60(95 \% \mathrm{CI}-0.60,7.79)$ and $5.32(95 \% \mathrm{CI}$ $-0.47,11.11$ ) additional life years gained, respectively, compared to the low PA group; corresponding values for 


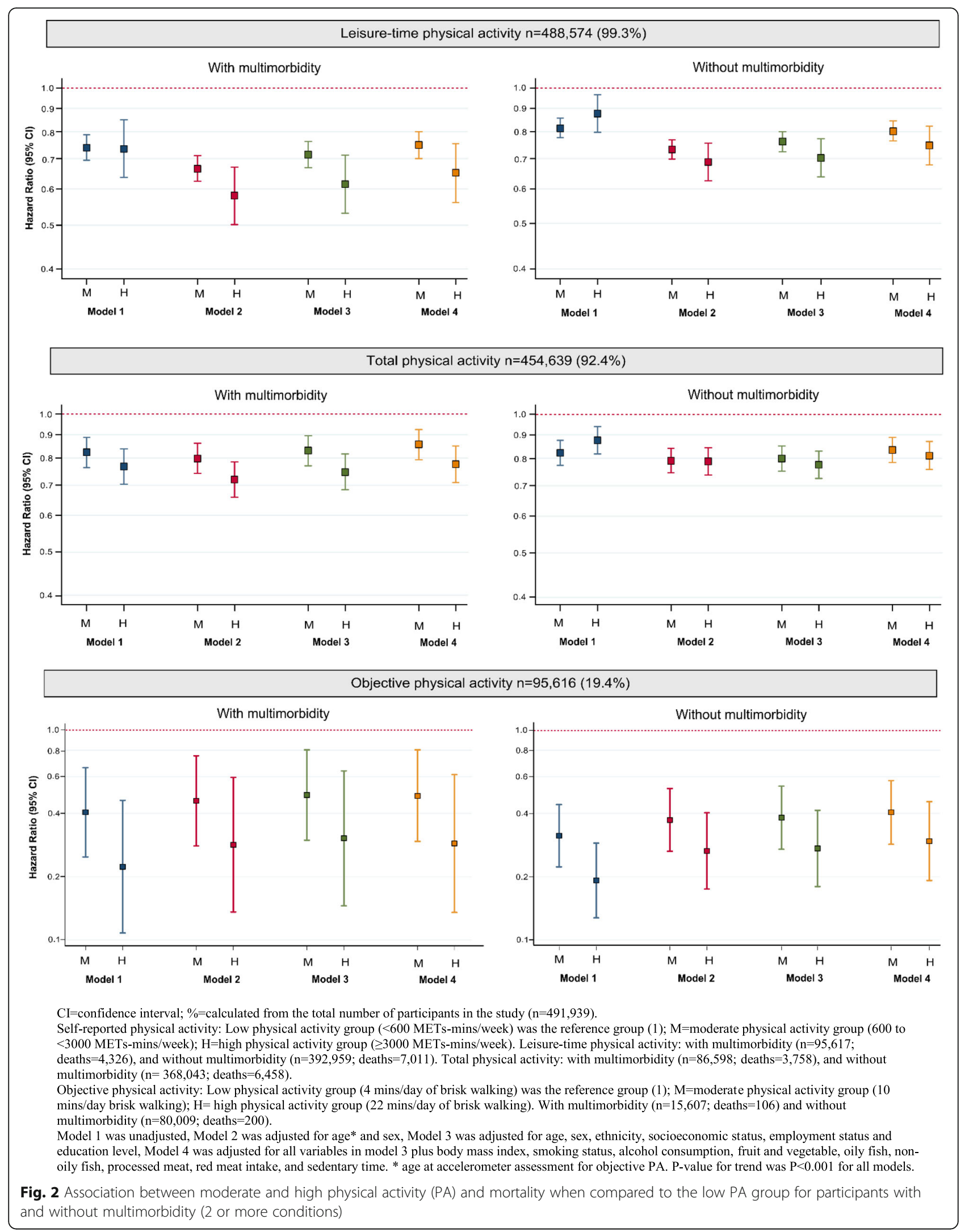




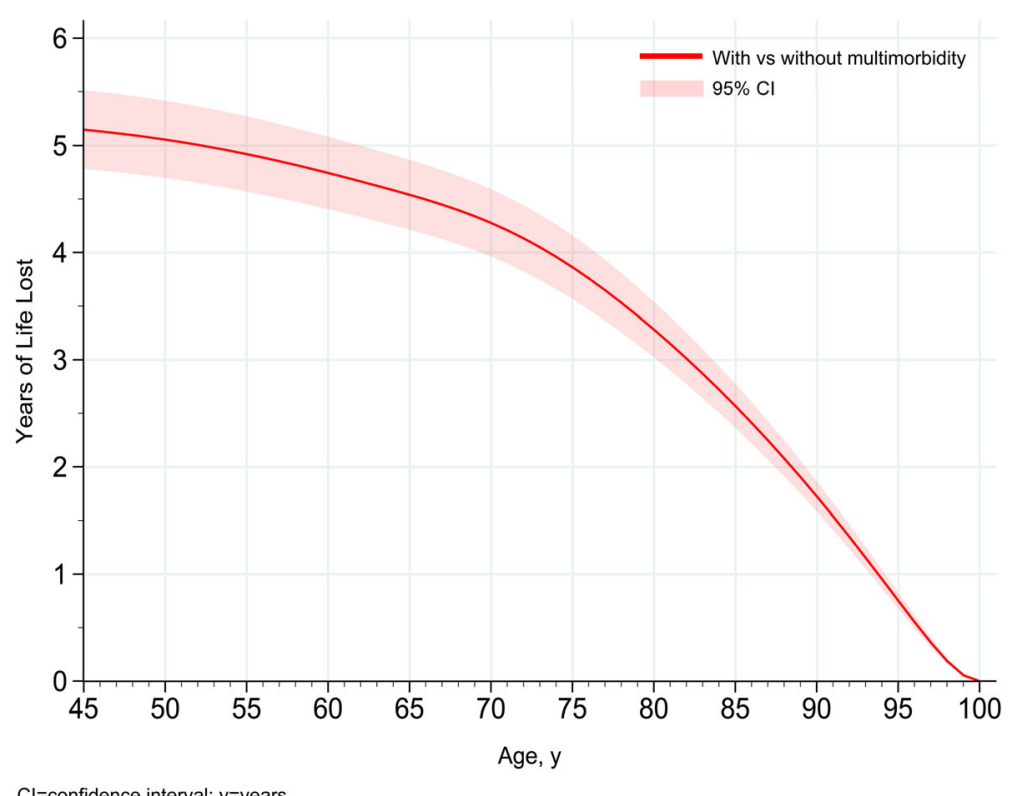

Fig. 3 Modelling years of life lost by multimorbidity (2 or more conditions)

those without multimorbidity were 3.88 (95\% CI 1.79 , $6.00)$ and 4.51 (95\% CI 2.15, 6.88) additional life years, respectively (Fig. 4). In sensitivity analyses, results were comparable when accounting for the severity of the conditions or top-10 comorbidity (Additional file 2: Tables S11 and S12).

\section{Discussion}

We found an inverse dose-response association between PA and mortality in participants with and without multimorbidity, which persisted even after adjustments for sociodemographic and lifestyle factors. For self-reported PA, achieving volumes of PA that are consistent with the current guidelines of $150 \mathrm{~min}$ of moderate-intensity PA per week was associated with a longer life expectancy, with little additional benefit seen above this amount. Moreover, results for objectively measured PA suggested as little as $10 \mathrm{~min}$ of brisk walking a day was associated with a longer life expectancy, again with little additional benefit seen at higher categories.

Only two studies have assessed the relationship between self-reported PA and mortality in people with multimorbidity. The first study from Spain $(n=3967)$, using LTPA, showed that in participants with multimorbidity $(n=571)$, being physically active was related to a $35 \%$ (95\% CI 16-50\%) lower risk of mortality, compared to being inactive [13]. Our results using LTPA mirror these findings, whereby there was a 35\% lower risk in the high PA group compared to the low PA group. In contrast, results from the analysis of the National Health and Nutrition Examination Survey dataset $(n=16,091)$ found that LTPA had a minimal effect on the multimorbidity- mortality relationship. The models were stratified by PA levels, and the number of chronic conditions was the exposure variable. Results showed that for each additional chronic condition, the association with mortality remained strongly significant, regardless of the amount of PA (i.e. stratifying by $\geq 4000$ MVPA MET-min/month indicated for each additional chronic condition, there was a $22 \%$ higher risk of mortality). However, for those who performed $\geq 8000 \mathrm{MET}$-min/month, the multimorbiditymortality relationship was much smaller (HR 1.08, 95\% CI $0.87-1.33$ ), suggesting that people with multimorbidity would gain a benefit only if they report extremely high levels of PA [14]. Other studies in large general populations support our findings, as they show that both moderate and vigorous-intensity activities were associated with a longevity benefit [32-34]. Although there are no studies that examine the comparative role of PA and multimorbidity on life expectancy, our results are in line with previous research based on different populations [12, 34-36].

This study has some limitations. Firstly, participation in the UK Biobank was voluntary with slightly higher representation from affluent groups; therefore, the results may not be completely representative of the UK population [37]. The sub-sample who undertook the objective PA measurements was relatively healthier than those who did not participate and was limited to only computer literate participants [38]; this limits the representativeness of the sample for objective PA analysis, and these results should be interpreted with caution. Another limitation is related to the time difference between the baseline assessment and objective PA assessment: we analysed and adjusted for covariates at 

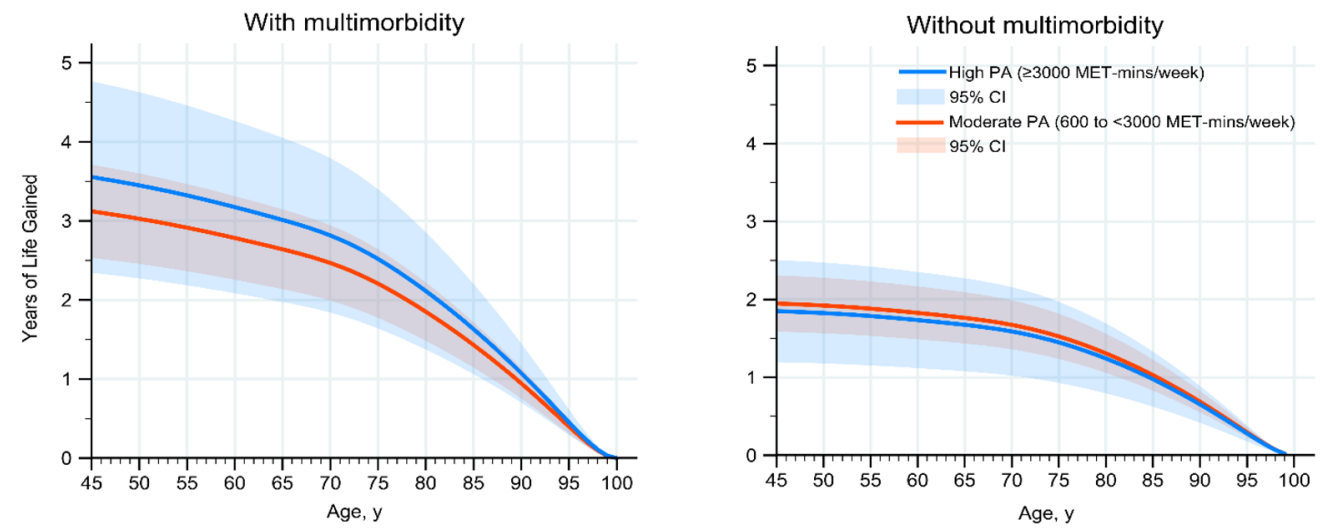

Total physical activity $n=454,639(92.4 \%)$
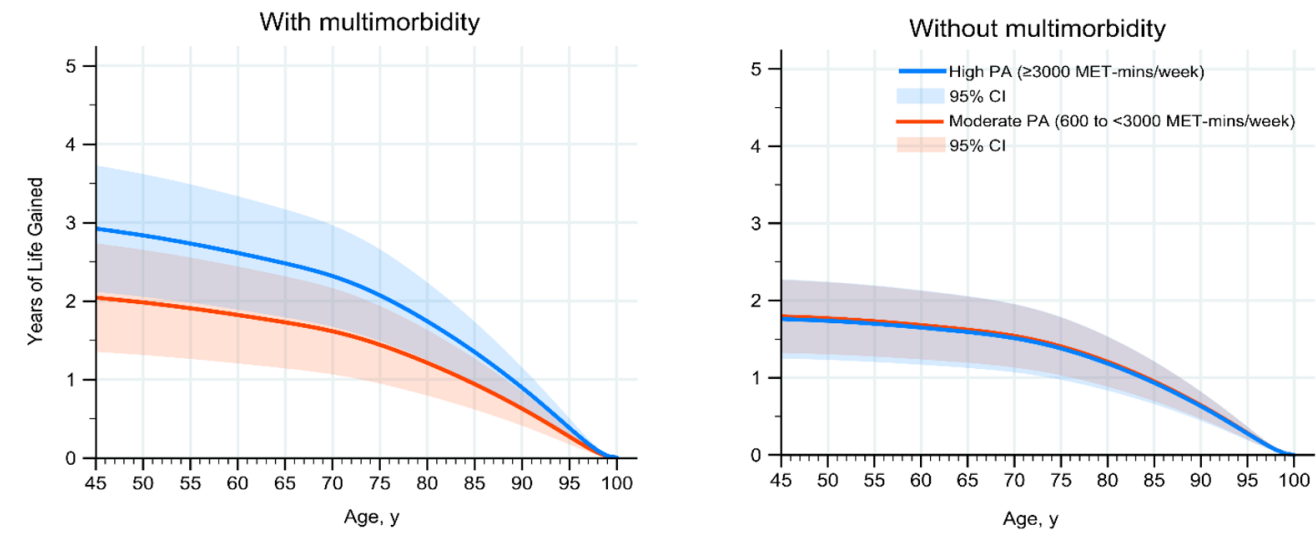

Objective physical activity $n=95,616(19.4 \%)$

With multimorbidity

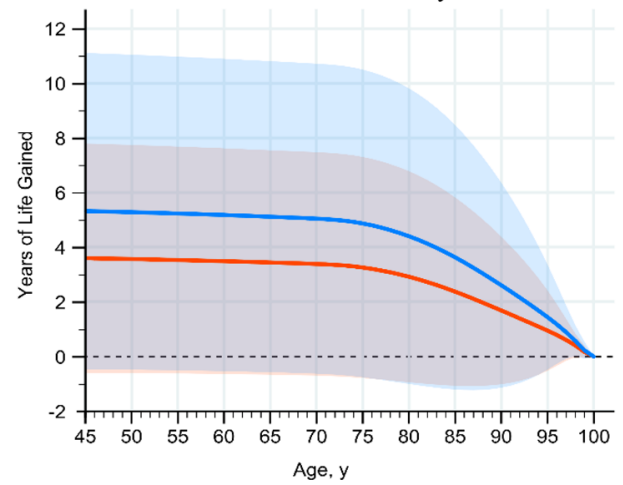

Without multimorbidity

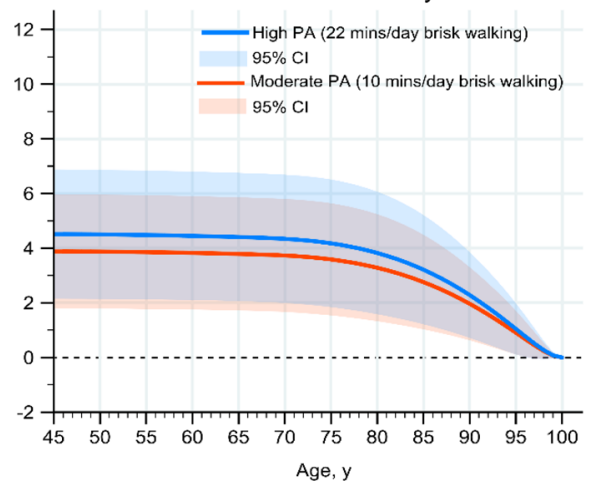

$\mathrm{PA}=$ physical activity; $\mathrm{CI}=$ confidence interval; $\mathrm{y}=\mathrm{years} ; \%=$ calculated from the total number of participants in the study $(\mathrm{n}=491,939)$. Leisure-time physical activity: With multimorbidity: Low PA ( $\mathrm{n}=53,658$; deaths $=2,743)$; Moderate PA $(\mathrm{n}=36,760$; deaths $=1,388)$; High PA $(\mathrm{n}=5,199$; deaths $=195)$. Without multimorbidity: Low PA $(\mathrm{n}=185,313$; deaths=3,652); Moderate PA $(\mathrm{n}=178,757$; deaths $=2,865) ;$ High PA $(\mathrm{n}=28,887$; deaths $=494)$.

Total physical activity: With multimorbidity: Low PA ( $\mathrm{n}=20,823$; deaths=1,063); Moderate PA ( $\mathrm{n}=43,120$; deaths $=1,811)$; High PA $(\mathrm{n}=22,655$; deaths $=884)$. Without multimorbidity: Low PA $(\mathrm{n}=69,875$; deaths=1,417); Moderate PA $(\mathrm{n}=189,736$; deaths $=3,142)$; High PA $(\mathrm{n}=108,430$; deaths $=1,899)$.

Objective physical activity: With multimorbidity: Low PA ( $n=7,369$; deaths=78); Moderate PA ( $n=4,757$; deaths=20); High PA ( $n=3,481$; deaths $=8$ ). Without multimorbidity: Low PA ( $\mathrm{n}=24,550$; deaths $=128)$; Moderate PA $(\mathrm{n}=27,114$; deaths $=44)$; High PA $(\mathrm{n}=28,345$; deaths=28).

Fig. 4 Life year gains associated with moderate and high physical activity (PA) when compared to the low PA group for participants with and without multimorbidity (2 or more conditions) 
baseline (i.e. shifting the objective PA backwards), thus assuming the PA levels of the participants did not change between baseline and objective PA assessment, which may not be true. Nevertheless, objective PA measures have been used in a similar fashion in another UK Biobank analysis [39]. Furthermore, data from a longitudinal study on older adults in England reported steady PA levels over time with a slight decline in the time spent in vigorous-intensity activities [40]. Therefore, our results may have slightly under-estimated the association between objective PA and mortality. However, the hazard ratio results remained unchanged when we used the time of objective PA assessment as the start of the follow-up. Moreover, the short follow-up for the cohort of participants with objective PA did not allow a landmark analysis excluding participants who died in the first 2 years. There is currently no standard method for measuring multimorbidity [41]; however, our definition included most of the core conditions for any multimorbidity measure $[7,23,25]$, and we have carried out sensitivity analyses using two different methods to ensure our findings are valid. Since this study was observational, we cannot derive any causality from the relationships we evidenced, yet there is substantial evidence supporting a causal relationship between higher levels of PA and good health status $[10,11,18,34,36]$. Finally, although we found an association between PA and higher life expectancy, especially in people with multimorbidity, we were unable to assess whether this translates to a better quality of life.

Strengths of this study include the large sample size used to assess the association between PA with mortality and life expectancy by multimorbidity status. Also, this is the first study to use objective PA to assess its association with mortality in people with multimorbidity. We controlled for a wide range of important confounders, including age, as this is related to both PA and multimorbidity; however, we cannot completely exclude the potential for residual confounding. We also removed deaths occurring within the first 2 years at baseline to minimise the risk of reverse causality. We used three different measures of PA and three different multimorbidity definitions to ensure that the findings are robust. When accounting for the severity of the conditions, we found that the message was even stronger for those with a poor health rating and multimorbidity, or with top-10 comorbidity, because the benefits of physical activity had a much greater impact on the years of life gained compared to those who had a good health state or without top-10 comorbidity.

\section{Conclusions}

In conclusion, our findings showed an inverse dose-response association between PA and all-cause mortality suggesting the mortality benefits of PA still apply in people with multimorbidity. Moreover, our results indicated that self-reported levels of physical activity that are consistent with the current physical activity guidelines, or as little as $10 \mathrm{~min}$ of brisk walking a day based on objective measurement, were associated with longer life expectancy with little additional benefit observed above these levels, implying that it is not necessary to engage in high-volume or high-intensity PA to achieve the potential health benefits. However, we identified a large number of participants with multimorbidity who did not reach this level of PA, suggesting that there is a need for individuals with multimorbidity to increase their PA in ways that are achievable.

\section{Additional files}

Additional file 1: Methods S1 List of the 36 chronic conditions included within the definition of multimorbidity. Methods S2 Physical activity measurements. Methods S3 Additional methods for sociodemographic and lifestyle factors. Methods S4 Summary of main and sensitivity analyses. (DOCX $31 \mathrm{~kb}$ )

Additional file $\mathbf{2}$ Table S1. Number of participants with comorbidity. Table S2. Number of participants by the total number of chronic conditions. Table S3. Most to least prevalent chronic conditions by overall health rating and multimorbidity status. Table S4. Participant characteristics by overall health status and multimorbidity. Table S5. Number of participants with the top-10 comorbidity. Table S6. Characteristics by participants with and without top-10 comorbidity. Table S7. Participant characteristics who undertook the objective physical activity by multimorbidity status. Table S8. Participants characteristics by completion of the objective physical activity measurements. Table S9. Self-reported physical activity in tertiles and years of life gained at the age of 45 years by multimorbidity status. Table S10. Association between continuously measured (log-transformed) physical activity and mortality. Table S11. Association between physical activity and mortality for participants with good or poor overall health rating and multimorbidity status, and years of life gained at the age of 45 years. Table S12. Association between physical activity and mortality for participants with and without top-10 comorbidity, and years of life gained at the age of 45 years. Table S13. Association between objective physical activity in tertiles of time accumulated above $250 \mathrm{mg}$ of total acceleration and mortality. Figure S1. Most to least prevalent chronic conditions used to define multimorbidity ( $n=491,939)$. Figure S2. Association between self-reported physical activity in tertiles and mortality. Figure S3. Association between objective physical activity and mortality, taking the time at objective PA assessment as the start of the follow-up in participants with and without multimorbidity. (DOCX 2092 kb)

\section{Abbreviations \\ A/AS Level: Advanced level qualifications; BMI: Body mass index; Cl: Confidence intervals; DIY: Do-it-yourself; GCSE: General certificate of secondary education; HR: Hazard ratio; IPAQ: International Physical Activity Questionnaire; IQR: Interquartile range; Log: Logarithm; LTPA: Leisure-time physical activity; MET: Metabolic equivalent; mg: Milli-gravitational units; MP: Multicore processor; MVPA: Moderate to vigorous physical activity; NHS: National Health Service; NVQ: National Vocational Qualification; PA: Physical activity; QoF: Quality and outcomes framework; TPA: Total physical activity; UK : United Kingdom}

Acknowledgements

This research has been conducted using the UK Biobank Resource (Reference 14614).

We acknowledge the support from the National Institute for Health Research Collaboration for Leadership in Applied Health Research and Care - East Midlands (NIHR CLAHRC - EM), the Leicester Clinical Trials Unit, and the NIHR 
Leicester Biomedical Research Centre. The views expressed are those of the authors and not necessarily those of the NHS, the NIHR, or the Department of Health.

As part of the $\mathrm{PhD}$, this study was selected to be presented at the following conferences: (1) CLAHRC East Midlands and North Thames Multimorbidity Collaboration Meeting 2017, Diabetes Research Centre, Leicester; (2) NIHR CLAHRC Cardiometabolic Research Day 2017, Stamford Court, Leicester; (3) Department of Health Sciences' Annual Conference 2018, Stamford Court, Leicester; (4) National NIHR CLAHRC Multimorbidity Research Event 2018, Stamford Court, Leicester; (5) Trent Region Society for Academic Primary Care 2018, St Marys Conference Centre, Sheffield (awarded prestigious prize for the best presentation); (6) Public Health Research and Science Conference 2018, Public Health England, Warwick University, Warwick; (7) European Diabetes Epidemiology Group Annual Meeting 2018, Hotel Marienlyst, Elsinore, Denmark; (8) Health Sciences Postgraduate Forum 2018 (HeSPoF), College Court, Leicester; (9) Public Health England Research and Development Meeting 2018, East Midlands Centre, Seaton House, Nottingham; and (10) The UK Biobank Scientific Conference 2018 (abstract placed within the top 20 in the UK Biobank Early Career Researcher of the Year competition), QEll Conference Centre, London.

\section{Funding}

YC is funded by a University of Leicester College of Medicine, Biological Sciences and Psychology PhD studentship in collaboration with Collaboration for Leadership in Applied Health Research and Care-East Midlands.

\section{Availability of data and materials}

The data that support the findings of this study are available from UK Biobank project site, subject to registration and application process. Further details can be found at https://www.ukbiobank.ac.uk.

Statistical codes for this study are available on GitHub (yc244) repository.

\section{Authors' contributions}

ND and KK conceived the idea of the study; ND acquired the data; YC carried out the statistical analysis; ND, KK, and YC interpreted the findings; and YC drafted the manuscript. YC had full access to all the data in the study and takes responsibility for the integrity of the data and the accuracy of the data analysis. FZ, AR, TY, MD, and CG provided input in the analysis; all authors critically reviewed the manuscript and $Y C$ revised the manuscript for final submission. All authors read and approved the final manuscript.

\section{Ethics approval and consent to participate}

All participants gave written informed consent prior data collection.UK Biobank has full ethical approval from the NHS National Research Ethics Service (16/NW/0274).

\section{Consent for publication}

Not applicable.

\section{Competing interests}

ND is currently a full-time employee of Evidera Inc. which is a contract research organisation providing research and consultancy support for pharmaceutical companies in using real-world data. FZ is a Clinical Research Fellow funded with an unrestricted Educational Grant from Sanofi-Aventis and an unrestricted educational grant from the NIHR CLAHRC East Midlands to the University of Leicester; the funding sources had no role in the study design, data collection, data analysis, data interpretation, or writing of the report. KK has acted as a consultant and speaker for Novartis, Novo Nordisk, SanofiAventis, Lilly, Servier, and Merck Sharp \& Dohme. He has received grants in support of investigator and investigator-initiated trials from Novartis, Novo Nordisk, Sanofi-Aventis, Lilly, Pfizer, Boehringer Ingelheim, and Merck Sharp \& Dohme. KK has received funds for research and honoraria for speaking at meetings and has served on advisory boards for Lilly, Sanofi-Aventis, Merck Sharp \& Dohme, and Novo Nordisk. MJD has acted as consultant, advisory board member, and speaker for Novo Nordisk, Sanofi-Aventis, Lilly, Merck Sharp \& Dohme, Boehringer Ingelheim, AstraZeneca, and Janssen and as a speaker for Mitsubishi Tanabe Pharma Corporation. She has received grants in support of investigator and investigator-initiated trials from Novo Nordisk, Sanofi-Aventis, and Lilly. All other authors declare there is no duality of interest in connection with their involvement in this study. All other authors declare no competing interests.

\section{Publisher's Note}

Springer Nature remains neutral with regard to jurisdictional claims in published maps and institutional affiliations.

\section{Author details}

${ }^{1}$ Diabetes Research Centre, Leicester General Hospital, University of Leicester, Leicester LE5 4PW, UK. ${ }^{2}$ National Institute for Health Research (NIHR) Collaboration for Leadership in Applied Health Research and Care - East Midlands (CLAHRC-EM), Leicester Diabetes Centre, Leicester LE5 4PW, UK. ${ }^{3} \mathrm{NIHR}$ Leicester Biomedical Research Centre, Leicester Diabetes Centre, Leicester LE5 4PW, UK. ${ }^{4}$ Alliance for Research in Exercise, Nutrition and Activity (ARENA), Sansom Institute for Health Research, Division of Health Sciences, University of South Australia, Adelaide, SA 5001, Australia. ${ }^{5}$ Current address: Real World Evidence, Evidera, London W6 8BJ, UK.

Received: 26 September 2018 Accepted: 1 May 2019

Published online: 12 June 2019

\section{References}

1. Kontis V, Bennett JE, Mathers CD, Li G, Foreman K, Ezzati M. Future life expectancy in 35 industrialised countries: projections with a Bayesian model ensemble. Lancet. 2017;389(10076):1323-35

2. National Institute for Health and Care Excellence: Multimorbidity: clinical assessment and management guidance and guidelines, 2016. Available at: https://www.nice.org.uk/guidance/ng56

3. Forman DE, Maurer MS, Boyd C, Brindis R, Salive ME, Horne FM, Bell SP, Fulmer T, Reuben DB, Zieman S. Multimorbidity in older adults with cardiovascular disease. J Am Coll Cardiol. 2018;71(19):2149-61.

4. Wallace E, Salisbury C, Guthrie B, Lewis C, Fahey T, Smith SM. Managing patients with multimorbidity in primary care. BMJ. 2015;350:h176.

5. Fortin M, Dubois M-F, Hudon C, Soubhi H, Almirall J. Multimorbidity and quality of life: a closer look. Health Qual Life Outcomes. 2007;5(1):52.

6. Lehnert T, Heider D, Leicht H, Heinrich S, Corrieri S, Luppa M, Riedel-Heller S, König $\mathrm{H}-\mathrm{H}$. Review: health care utilization and costs of elderly persons with multiple chronic conditions. Med Care Res Rev. 2011;68(4):387-420.

7. Barnett K, Mercer SW, Norbury M, Watt G, Wyke S, Guthrie B. Epidemiology of multimorbidity and implications for health care, research, and medical education: a cross-sectional study. Lancet. 2012; 380(9836):37-43.

8. Tinetti ME, Fried TR, Boyd CM. Designing health care for the most common chronic condition-multimorbidity. JAMA. 2012;307(23):2493-4.

9. The Academy of Medical Sciences. Multimorbidity: a priority for global health research; 2018. p. 127. Available at: https://acmedsci.ac.uk/filedownload/82222577

10. Kokkinos P. Physical activity, health benefits, and mortality risk. ISRN Cardiol. 2012;2012:718789

11. Warburton DE, Nicol CW, Bredin SS. Health benefits of physical activity: the evidence. Can Med Assoc J. 2006;174(6):801-9.

12. Li Y, Pan A, Wang DD, Liu X, Dhana K, Franco OH, Kaptoge S, Di Angelantonio E, Stampfer M, Willett WC, et al. Impact of healthy lifestyle factors on life expectancies in the US population. Circulation. 2018;138(4):345-55.

13. Martinez-Gomez D, Guallar-Castillon P, Garcia-Esquinas E, Bandinelli S, Rodríguez-Artalejo F. Physical activity and the effect of multimorbidity on all-cause mortality in older adults. In: Mayo Clinic Proceedings: 2017. Elsevier; 2017. p. 376-82.

14. Loprinzi PD, Addoh O, Joyner C. Multimorbidity, mortality, and physical activity. Chronic Illness. 2016;12(4):272-80.

15. Shephard RJ. Limits to the measurement of habitual physical activity by questionnaires. Br J Sports Med. 2003;37(3):197-206.

16. Barreto PS. Global health agenda on non-communicable diseases: has WHO set a smart goal for physical activity? BMJ. 2015;350:h23.

17. Prince SA, Adamo KB, Hamel ME, Hardt J, Gorber SC, Tremblay M. A comparison of direct versus self-report measures for assessing physical activity in adults: a systematic review. Int J Behav Nutr Phys Act. 2008;5(1):56.

18. Sparling PB, Howard BJ, Dunstan DW, Owen N. Recommendations for physical activity in older adults. BMJ: Br Med J. 2015:350. 
19. UK Biobank Coordinating Centre, UK Biobank: Protocol for a large-scale prospective epidemiological resource, 2017, Available at: [http://www. ukbiobank.ac.uk/wp-content/uploads/2011/11/UK-Biobank-Protocol.pdf].

20. UK Biobank, UK Biobank physical activity monitor (accelerometer), version 1. 0, 2016, Available at: (https:/biobank.ctsu.ox.ac.uk/crystal/docs/ PhysicalActivityMonitor.pdf).

21. UK Biobank. UK Biobank ethics and governance framework 2007. Available at https://www.ukbiobank.ac.uk/wp-content/uploads/2011/05/EGF20082.pdf. Accessed 12 Feb 2018.

22. UK Biobank. Access procedures: application and review procedures for access to the UK Biobank resource, version 1.0. Stockport: UK Biobank Coordinating Centre; 2011.

23. Zemedikun DT, Gray LJ, Khunti K, Davies MJ, Dhalwani NN. Patterns of multimorbidity in middle-aged and older adults: an analysis of the UK Biobank Data. Mayo Clin Proc. 2018:93(7):857-66

24. Doran T, Kontopantelis E, Reeves D, Sutton M, Ryan AM. Setting performance targets in pay for performance programmes: what can we learn from QOF? BMJ. 2014;348:g1595.

25. Diederichs C, Berger K, Bartels DB. The measurement of multiple chronic diseases - a systematic review on existing multimorbidity indices. J Gerontol Ser A Biol Med Sci. 2011;66(3):301-11.

26. The IPAQ Group, IPAQ scoring protocol - International Physical Activity Questionnaire. Available at: https://sites.google.com/site/theipaq/scoringprotocol. Accessed 26 Mar 2018.

27. Hildebrand MVHV, Hansen BH, Ekelund U. Age-group comparibility of raw accelerometer output from wrist- and hip-worn monitors. Med Sci Sports Exerc. 2014;46(9):1816-24.

28. Dehbi H-M, Royston P, Hackshaw A. Life expectancy difference and life expectancy ratio: two measures of treatment effects in randomised trials with non-proportional hazards. BMJ. 2017;357:j2250.

29. Nelson CP, Lambert PC, Squire IB, Jones DR. Flexible parametric models for relative survival, with application in coronary heart disease. Stat Med. 2007;26(30):5486-98

30. Lambert PC, Royston P. Further development of flexible parametric models for survival analysis. Stata J. 2009;9(2):265.

31. Guthrie B, Payne K, Alderson P, McMurdo ME, Mercer SW. Adapting clinical guidelines to take account of multimorbidity. BMJ. 2012;345:e6341.

32. Arem H, Moore SC, Patel A, et al. Leisure time physical activity and mortality: a detailed pooled analysis of the dose-response relationship. JAMA Intern Med. 2015;175(6):959-67.

33. Lee IM, Paffenbarger RS Jr. Associations of light, moderate, and vigorous intensity physical activity with longevity. The Harvard Alumni Health Study. Am J Epidemiol. 2000;151(3):293-9.

34. Wen CP, Wai JP, Tsai MK, Yang YC, Cheng TY, Lee MC, Chan HT, Tsao CK, Tsai SP, Wu X. Minimum amount of physical activity for reduced mortality and extended life expectancy: a prospective cohort study. Lancet. 2011;378(9798):1244-53.

35. Franco $\mathrm{OH}, \mathrm{Cd} \mathrm{L}$, Peeters $\mathrm{A}$, Jonker J, Mackenbach J, Nusselder W. Effects of physical activity on life expectancy with cardiovascular disease. Arch Intern Med. 2017:165(20):2355-60.

36. Reimers $C D$, Knapp G, Reimers AK. Does physical activity increase life expectancy? A review of the literature. J Aging Res. 2012;2012:9.

37. Swanson JM. The UK Biobank and selection bias. Lancet. 2012;380(9837):110.

38. Doherty A, Jackson D, Hammerla N, Plötz T, Olivier P, Granat MH, White T, van Hees VT, Trenell Ml, Owen CG. Large scale population assessment of physical activity using wrist worn accelerometers: the UK Biobank study. PLoS One. 2017;12(2):e0169649.

39. Stiles VH, Metcalf BS, Knapp KM, Rowlands AV. A small amount of precisely measured high-intensity habitual physical activity predicts bone health in pre-and post-menopausal women in UK Biobank. Int J Epidemiol. 2017:46(6):1847-56.

40. Smith L, Gardner B, Fisher A, Hamer M. Patterns and correlates of physical activity behaviour over 10 years in older adults: prospective analyses from the English Longitudinal Study of Ageing. BMJ Open. 2015;5(4):e007423.

41. Le Reste JY, Nabbe P, Manceau B, Lygidakis C, Doerr C, Lingner H, Czachowski S, Munoz M, Argyriadou S, Claveria A. The European General Practice Research Network presents a comprehensive definition of multimorbidity in family medicine and long term care, following a systematic review of relevant literature. J Am Med Dir Assoc. 2013;14(5):319-25.

\section{Ready to submit your research? Choose BMC and benefit from:}

- fast, convenient online submission

- thorough peer review by experienced researchers in your field

- rapid publication on acceptance

- support for research data, including large and complex data types

- gold Open Access which fosters wider collaboration and increased citations

- maximum visibility for your research: over $100 \mathrm{M}$ website views per year

At $\mathrm{BMC}$, research is always in progress.

Learn more biomedcentral.com/submissions 